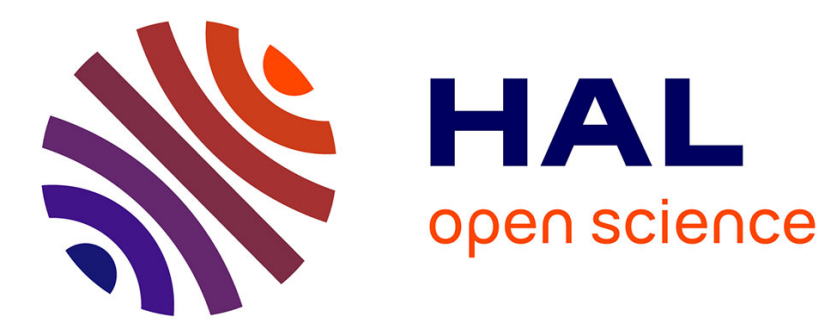

\title{
Quand le territoire joue: une comparaison de la prise en compte des enjeux sociaux-spatiaux dans les politiques intercommunales de transports publics
}

\author{
Martin Claux
}

\section{- To cite this version:}

Martin Claux. Quand le territoire joue: une comparaison de la prise en compte des enjeux sociauxspatiaux dans les politiques intercommunales de transports publics. RTS - Recherche Transports Sécurité, 2013, 2013 (01), pp.5-20. 10.4074/S0761898013001027 . hal-00942673

\section{HAL Id: hal-00942673 \\ https://hal.science/hal-00942673}

Submitted on 15 Mar 2018

HAL is a multi-disciplinary open access archive for the deposit and dissemination of scientific research documents, whether they are published or not. The documents may come from teaching and research institutions in France or abroad, or from public or private research centers.
L'archive ouverte pluridisciplinaire HAL, est destinée au dépôt et à la diffusion de documents scientifiques de niveau recherche, publiés ou non, émanant des établissements d'enseignement et de recherche français ou étrangers, des laboratoires publics ou privés. 


\title{
Quand le territoire joue : \\ une comparaison de la prise en compte des enjeux sociaux-spatiaux dans les politiques intercommunales de transports publics
}

\author{
When the territory count: A comparison of the socio-spatial issue consideration in the \\ intermunicipal public transport policies
}

\author{
Martin Claux
}

Reçu le 8 juin 2012 ; accepté le 22 décembre 2012

(C) IFSTTAR et Éditions NecPlus 2013

Résumé À travers la déconstruction des critères qui président à l'organisation des réseaux de deux politiques intercommunales de transports et de déplacements, cet article vise à apporter une contribution à la question $\mathrm{du}$ sens de l'action publique urbaine en s'écartant des discours convenus sur le développement urbain durable. Méthodologiquement, nous étudions l'identité socio-économique de ces territoires pour ensuite les confronter aux choix différenciés d'organisation des réseaux de transports. L'organisation du réseau de transport est appréhendée en premier lieu par une spatialisation des territoires qui bénéficient du déploiement de la desserte intercommunale en transport public puis dans un second temps par une confrontation de ces résultats aux discours de légitimation et de justification tenus par les acteurs de ces politiques urbaines. Cet article aborde donc les ambitions différenciées des politiques intercommunales de transport et de déplacements avec pour hypothèse centrale que l'identité économique et sociale des territoires intercommunaux influence fortement les stratégies de politiques publiques en matière de transport et de déplacements.

Mots clés solidarité socio-spatiale · intercommunalités . transports et déplacements · région urbaine marseillaise . entrepreneurialisme

L'auteur remercie les responsables, tout particulièrement Hélène Reigner, et les participants au projet Soliter, du programme Gouverner et administrer de l'ANR, ainsi que les deux relecteurs/relectrices anonymes de la revue pour leurs discussions des précédentes versions de ce texte.

Martin Claux $(\square)$

Institut d'Urbanisme et d'Aménagement Régional, Aix-Marseille Univ., France, IFSTTAR, TS2/LMA, 304 Chemin de la Croix Blanche F-13300 Salon-de-Provence

e-mail : martin.claux@gmail.com

\begin{abstract}
Through the observation of criteria witch are involved in the organization of the public transport system, this paper contribute to define the meaning of urban public policies while going away from the conventional discourse about sustainable development. Formaly we are studying the socio-economic identity of two territory in order to confront the different choice made in the public transport organization. First, the public transports network organization is grasping through the spatialization of the territory which benefit by the deployment of the intercommunal bus service. Second, this results, and theirs maps, are confront to the legitimation and justification discourse made by the actors of these policies. Finaly, this article approaches the different ambitions of the public transport intercommunal policies with the central hypothesis that the socio-economic identity of the territory plays a major role in the strategies deployed by the intercommunal level of government.
\end{abstract}

Keywords Socio-spatial solidarity · Intercommunal · public transport - Marseille urban area .

entrepreneurialism

\section{Introduction}

La montée en puissance des structures intercommunales d'agglomération fait des intercommunalités une arène où se décident collectivement les trajectoires urbaines, mais le caractère démocratique de ces arènes intercommunales et des politiques publiques produites en leur sein fait débat [1]. En matière de transport et de déplacements, le sens des politiques communautaires est aussi posé [2], d'autant plus qu'un discours convenu sur les problématiques de déplacements se généralise [3]. Certes, toutes les intercommunalités communiquent abondamment sur leurs 
politiques de transport et de déplacements et vantent les effets (supposés) durables de leurs politiques exemplaires [4]. Cependant, au-delà de l'exercice de communication, toutes ces politiques s'engagent-elles dans une tentative de régulation durable des mobilités urbaines, c'est-à-dire dans la maitrise sur le temps long des conséquences sociales et environnementales de la mobilité ? Il ne s'agit pas dans cet article, de procéder à une évaluation des conséquences environnementales des politiques de déplacements mais d'interroger les politiques intercommunales de transport et de déplacements sous l'angle de leurs effets sociospatiaux. Cet intérêt pour la focale sociale est justifié à double titre.

Premièrement, la mobilité spatiale est liée à la mobilité sociale. Dans une société de plus en plus réticulaire où la connectivité prend le pas sur la contiguïté, le potentiel de mobilité des individus représente un vecteur de différenciation des trajectoires sociales, qui prend d'autant plus d'importance que la mobilité spatiale est socialement valorisée [5]. Dans cet environnement urbain, le potentiel de mobilité des pauvres est bien différent de celui dont disposent les riches : ils ont moins accès au volant, leur mobilité est davantage contrainte, elle leur offre moins d'opportunités et les ancre, voire les assigne, dans leurs territoires de résidences [6]. L'urbanité de ces populations modestes est contrainte, ce qui grève leur capacité à se mouvoir dans l'espace social ; que ces populations habitent les quartiers populaires des villes [6] ou encore le périurbain lointain [7]. La prise en compte de cette focale sociale de la mobilité par les politiques locales de déplacements représente une opportunité d'améliorer le potentiel de mobilité de ces populations. Or, on sait que l'accès aux réseaux de transport public, qui est pourtant un élément primordial du potentiel de mobilité spatiale des pauvres, tend à profiter à certains groupes sociaux au détriment d'autres, notamment en raison des contraintes de prix qui pèsent sur la localisation résidentielle des ménages populaires et de la dispersion dans les périphéries des activités qui emploient ces populations [8,9]. En fait, la question sociale de la mobilité reste encore confinée aux politiques sociales $[6,10]$. Dans le domaine du transport et des déplacements, cette question est l'angle mort des politiques locales. [11].

Deuxièmement, il est possible de dépasser ces constats et d'aller plus loin en proposant une interprétation des politiques urbaines empruntant à des cadres d'analyse radicaux issus de la géographie néo-marxiste [12]. Délaissant l'objectif de redistribution, les politiques urbaines contemporaines témoigneraient d'un tournant entrepreneurial : elles auraient pour objectif central d'attirer et de retenir sur le territoire des capitaux, des entreprises et des populations solvables « créatives », considérés comme les moteurs de la croissance économique et de la compétitivité urbaine [13]. Pour cela, et dans un contexte de compétition interurbaine accrue, concentrer les investissements publics pour créer de la valeur foncière et immobilière serait devenu une stratégie explicite des gouvernements urbains [14]. Dans ce contexte, la qualité urbaine, objectif récurrent des politiques intercommunales de transport et de déplacements, serait un attribut nécessaire de la métropole attractive pour figurer au palmarès des « villes où il fait bon vivre ». Parallèlement à cet enjeu de qualité urbaine dans les centres urbains historiques et patrimoniaux, les agglomérations poursuivent néanmoins l'amélioration de l'accessibilité routière à l'échelle de l'agglomération : polariser la croissance et le développement signifie aussi limiter les coûts de congestion par une bonne connection aux flux interurbains, interrégionaux voir internationaux grâce à des réseaux routiers rapides et performants. Ainsi, en dépit d'une planification qui fait ses gros titres sur le développement durable, les politiques déployées ne chercheraient donc pas tant à freiner globalement les mobilités polluantes qu'à les canaliser sur des infrastructures routières de contournement pour en limiter les impacts négatifs dans les hauts lieux de la ville $[15,16]$. Les nuisances automobiles (bruit, congestion, pollution, insécurité) seraient traitées comme des risques qu'il faut limiter et écarter de certains micro-territoires urbains stratégiques et symboliques.

Ainsi, les politiques publiques intercommunales de transport et de déplacements façonneraient des réseaux inégalement distribués sur les territoires des agglomérations, en vue de produire de la valorisation foncière et immobilière, et ne parviendraient pas à diminuer les inégalités d'accès à la ville. Cette inégale distribution spatiale de la desserte mérite d'être questionnée sous l'angle de ses impacts socio-économiques. L'attraction des moteurs de la croissance urbaine, par l'amélioration de la qualité de vie et la concentration des investissements publics en un lieu, prend-elle le pas sur la dimension sociale de la mobilité quotidienne dans les politiques intercommunales de transport et de déplacements ? La dimension socio-spatiale de la mobilité quotidienne est-elle pensée, intégrée dans ces politiques ? Comment et pour faire quoi ? Finalement, dans un contexte «d'homogamie des mariages intercommunaux » [17] n'existe-t-il pas des variables territoriales qui participent à différencier la prise en compte de la dimension socio-spatiale de la mobilité quotidienne?

C'est pour répondre à ces questions que nous avons cherché à évaluer les politiques intercommunales à travers les territoires et groupes sociaux qui gagnent et qui perdent aux choix effectués dans l'organisation des réseaux de transport. La thèse que nous défendons est la suivante : 
l'histoire socio-économique des territoires étudiés est une variable structurante de la compréhension des stratégies de politiques publiques déployées par les gouvernements intercommunaux et de la compréhension des territoires et groupes sociaux gagnants et perdants. Selon nous, les différences observées dans la composition sociale du territoire et la structure urbaine jouent et participent à la différenciation territoriale des politiques intercommunales de transports et de déplacements.

Notre démonstration s'effectue en trois temps et l'on recourt à un outillage méthodologique original (Encadré1) ${ }^{1}$. Tout d'abord, nous nous intéressons à l'histoire institutionnelle et socio-économique des territoires étudiés. Situées toutes deux dans la même région urbaine (Figure 2), ces intercommunalités ont de fortes différences socioéconomiques et institutionnelles que nous mettons en avant. Ensuite, dans un second et un troisième temps de la démonstration, on s'attache plus particulièrement à la substance des politiques intercommunales de transports et de déplacements à travers les critères de décisions et les choix effectués dans l'organisation des réseaux de transports publics. On s'intéresse alors à la distribution spatiale de la desserte en transports publics, à la cartographie des espaces gagnants de la politique communautaire, tout en confrontant ces résultats aux dires des édiles intercommunaux. Finalement, nous affinons notre appréhension de la substance de ces politiques en comparant deux projets symboliques portés à l'agenda par les présidents de ces intercommunalités.

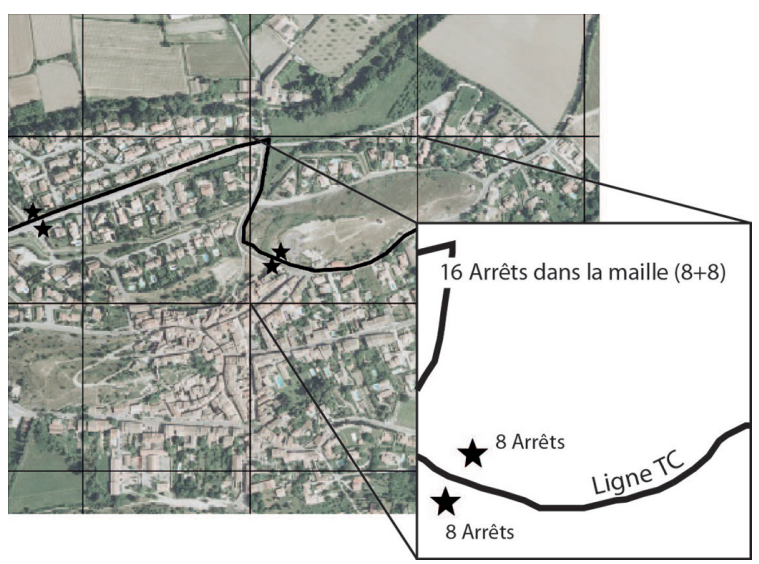

Fig. 1 Construction de l'indice de densité de desserte

\footnotetext{
${ }^{1}$ Cet article s'appuie sur un travail de thèse en cours portant sur la planification des politiques intercommunales de déplacements des pôles urbains intermédiaires de la Région Urbaine Marseillaise.
}

\section{Encadré 1. Une méthodologie originale}

Notre démarche associe méthodologies quantitative et qualitative. Les politiques intercommunales de déplacements présentées dans cet article ont été étudiées à travers le traitement de données statistiques, la spatialisation de la desserte en transports publics, le dépouillement d'un corpus de documents ${ }^{2}$ et la réalisation d'entretiens ${ }^{3}$.

Les éléments empiriques présentés dans la partie relative à l'histoire institutionnelle et socio-économique des territoires intercommunaux, sont issus de sources variées et systématiquement confrontées : récits produits par les édiles intercommunaux lors des entretiens, étude de la littérature grise, caractéristiques sociales des populations résidentes ainsi que leurs caractéristiques de mobilités (recensement harmonisé de la population 1968-2007 ; fichier détails Migcom et Migpro de l'Insee).

La cartographie de la distribution spatiale de la desserte en transport public présentée dans cet article est construite en " jetant un filet » sur le territoire communautaire, puis en relevant pour chaque maille de ce filet le nombre d'arrêts qu'y effectuent les transports collectifs (Figure 1). Chaque maille est un carré de 250 mètres de côté. Ensuite, ces cartes représentent un support d'échange, de discussion lors des entretiens.

Les deux projets symboliques étudiés ont été réalisés et mis en œuvre au cours des deux dernières années. Ils sont présentés en ce qu'ils symbolisent les tendances dominantes de ces deux politiques communautaires. En effet, ils sont tous deux une commande politique émanant des présidents des intercommunalités et sont présentés par les édiles intercommunaux comme des étapes importantes dans l'évolution de la politique communautaire. Ces projets sont étudiés à travers la communication institutionnelle qui est produite à leur sujet et la réalisation d'entretiens auprès des techniciens et élus impliqués dans ces projets.

\footnotetext{
${ }^{2}$ Archives des délibérations communautaires et communales, presse locale, documents de planification stratégique (Schéma de Cohérence Territoriale, Plan de Déplacements Urbains, Plan Locaux de l'Habitat) et journaux communaux et intercommunaux de communication.

${ }^{3}$ Une vingtaine d'entretiens semi-directifs a été réalisée auprès des acteurs de l'intercommunalité, des services techniques des villes de ces intercommunalités, de l'État local et du Syndicat Mixte des Transport de l'Est de l'Étang de Berre.
} 


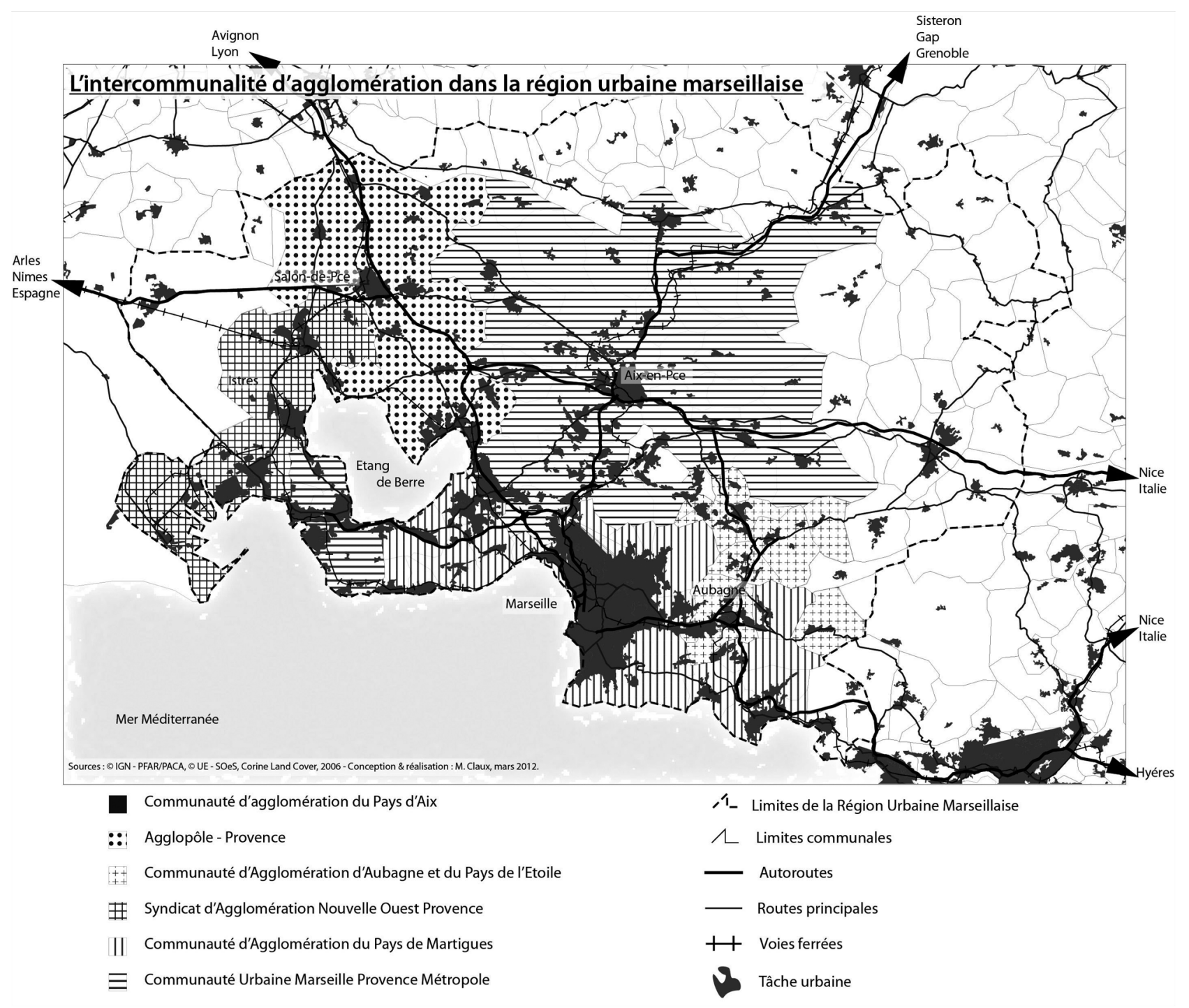

Fig. 2 Région Urbaine Marseillaise, situation

\section{Un « club plaqué or $»^{4}$ et un « territoire rouillé $»^{5}$ de la région urbaine marseillaise}

La Région urbaine marseillaise (RUM) est un territoire fragmenté à bien des égards. Économiquement, tandis que le centre périclitait jusqu'à la fin de la décennie 1990, les

\footnotetext{
${ }^{4}$ Ce terme est inspiré de la caractérisation des régimes intercommunaux que l'on doit à Philippe Estèbe dans son ouvrage Gouverner la ville mobile. Intercommunalité et démocratie locale, PUF, 2008, 80 p.

${ }^{5}$ Le terme « rouillé » renvoie à la géographie économique et plus précisément au terme rust belt qui défini l'espace industriel en déprise du nord-est des États-Unis d'Amérique.
}

périphéries accueillaient un dynamisme économique sans précédent. Politiquement, la coopération intercommunale est récente et reste encore prudente. Socialement, Marseille et sa région urbaine apparaissent comme l'un des territoires urbains les plus inégalitaires de France, après l'Île de France. À l'échelle métropolitaine, les deux territoires étudiés n'ont pas les mêmes caractéristiques. La Communauté d'agglomération agglopole provence (CA-AP) est rarement considérée comme un pôle moteur de la construction métropolitaine, c'est un satellite résidentiel. Quant au Syndicat d'agglomération nouvelle Ouest-Provence (SAN-OP), la présence du Grand port maritime de Marseille en fait un pôle industriel de la région urbaine, c'est un territoire majeur de l'actuel système productif marseillais. 


\section{La CA-AP : un territoire "plaqué or », une politique de déplacement dominée par la ville centre}

Aucune logique ne destinait les 17 communes de la communauté d'agglomération à s'engager dans la construction d'une structure intercommunale. À la veille des années 2000, trois structures intercommunales regroupant la majeure partie de ces 17 communes vont engager des discussions en vue d'élaborer une communauté d'agglomération. Ce ne sera «pas un mariage d'amour, mais un mariage d'intérêt [où] l'intérêt du mariage est financier $»^{6}$ qui s'effectuera sous le signe de la défiance respective des trois blocs intercommunaux préexistants : la communauté de communes collines Durance au nord, le district du pays salonais au centre et le district du multipôle de l'Étang de Berre au sud. Ce climat de défiance fait de cette intercommunalité une arène dominée par les communes et par les anciens territoires intercommunaux. Ces trois espaces, répartis sur un axe nord-sud, sont toujours distingués au sein des documents de planification stratégique (Figure 5). Au sud, se trouve un territoire industriel et ouvrier. Au centre, la « Provence salonaise » est un territoire "socialement doré », où le desserrement démographique en provenance du Pays d'Aix est haut de gamme. Au nord, l'ancienne plaine agricole de la Basse-Durance se transforme depuis plus de vingt ans en un périurbain lointain et populaire de la Région urbaine marseillaise.

Dans cette intercommunalité, le tandem Berre-l'Etang/Salon-de-Provence travaille à maintenir une structure considérée comme fragile. Le sénateur-maire de Berre-1'Étang, Serge Andréoni, est une personnalité locale du Parti socialiste et l'homme fort de l'intercommunalité. Michel Tonon, ancien rugbyman professionnel reconvertit dans la politique, est maire apparenté socialiste de Salon-de-Provence depuis 2002 dans une commune traditionnellement de centre droit. Ce tandem gère une « intercommunalité de tuyau » dont les compétences sont volontairement limitées à la prise en charge d'un triptyque de grands services : eau et assainissement, gestion des déchets et transports. Salon-de-Provence exerce un leadership certain sur cette dernière compétence. Cette position dominante de Salon-de-Provence est issue d'un soutien constant de Berre-l'Étang et des communes $\mathrm{du}$ sud de l'intercommunalité dans l'organisation du réseau communautaire. Ce soutien des communes du sud n'est en fait qu'un échange de bons procédés vis-à-vis d'une institution qui a montré sa détermination dans la préservation d'un réseau de transports collectifs au sud de l'intercommunalité.

\footnotetext{
${ }^{6}$ Entretien, directeur à l'urbanisme de la CA-AP, mai 2009.
}

En effet, la CA-AP n'organise pas la desserte en transports urbains sur le sud de son territoire et, depuis 1990, trois communes du sud de l'intercommunalité, dont Berre-l'Étang, appartiennent au Syndicat intercommunal de transport de l'est de l'Étang de Berre (Siteeb), créée en 1982 par Vitrolles et Marignane. Or, suite à la construction des nouveaux territoires intercommunaux au début des années 2000 (CA-AP, CA du Pays d'Aix, $\mathrm{CU}$ de Marseille), le réseau de transports collectifs du Siteeb court le risque d'être partitionné puisqu'il est éclaté entre différents territoires intercommunaux. Tous ces nouveaux partenaires ne souhaitent pas conserver l'unité de ce réseau. Par exemple, la communauté du Pays d'Aix, premier contributeur financier du Siteeb, est réticente à l'idée de coopérer avec les autres Autorités organisatrices des transports (intercommunalités et conseil général) et a menacé à plusieurs reprises d'éclater la gestion du réseau $\mathrm{du}$ Siteeb entre les différents partenaires (CA-AP, CA du Pays d'Aix, CU de Marseille et CG 13). Ce faisant, la communauté du Pays d'Aix rendait l'avenir du Siteeb incertain. Finalement, le Siteeb sera transformé en un Syndicat mixte des transport de l'est de l'Étang de Berre (Smiteeb), chargé d'organiser le réseau de transport public sur son territoire ${ }^{7}$. La CA du Pays d'Aix s'est finalement ralliée à cette solution institutionnelle.

Pendant la période durant laquelle l'avenir du Siteeb était incertain, la CA-AP a largement participé à la conservation de ce réseau ${ }^{8}$. Ainsi, le vice-président de la CA-AP délégué aux transports est le maire de Velaux ${ }^{9}$ et l'actuel président du Smiteeb. De plus, le chef de projet du Plan de déplacements urbains de la CA-AP est le directeur du Siteeb puis du Smiteeb. Ce soutien apporté par l'intercommunalité aux communes du sud afin qu'elles conservent leur réseau et qu'elles gardent la main sur son organisation - engendre une réciprocité : les élus de Berre l'Étang et ceux du sud soutiennent d'une seule voix le développement du réseau communautaire. De plus, Salon-de-Provence s'assure le soutien de Berrel'Étang quant au développement du réseau communautaire à travers d'autres compétences : la piscine de Berrel'Étang est un équipement communautaire, contrairement à celle de Salon-de-Provence qui reste sous le giron municipal.

\footnotetext{
${ }^{7}$ Dans les faits, ce Syndicat mixte de type SRU fonctionne comme une Autorité organisatrice des transports urbains à part entière.

${ }^{8}$ La CA-AP n'a pas œuvré seule au maintien de la structure, le Conseil général des Bouches-du-Rhône et le délégataire de service public se sont aussi mobilisés pour transformer le Siteeb en un syndicat mixte.

${ }^{9}$ Velaux est l'une des trois communes de l'intercommunalité desservie par le réseau du Siteeb puis du Smiteeb.
} 


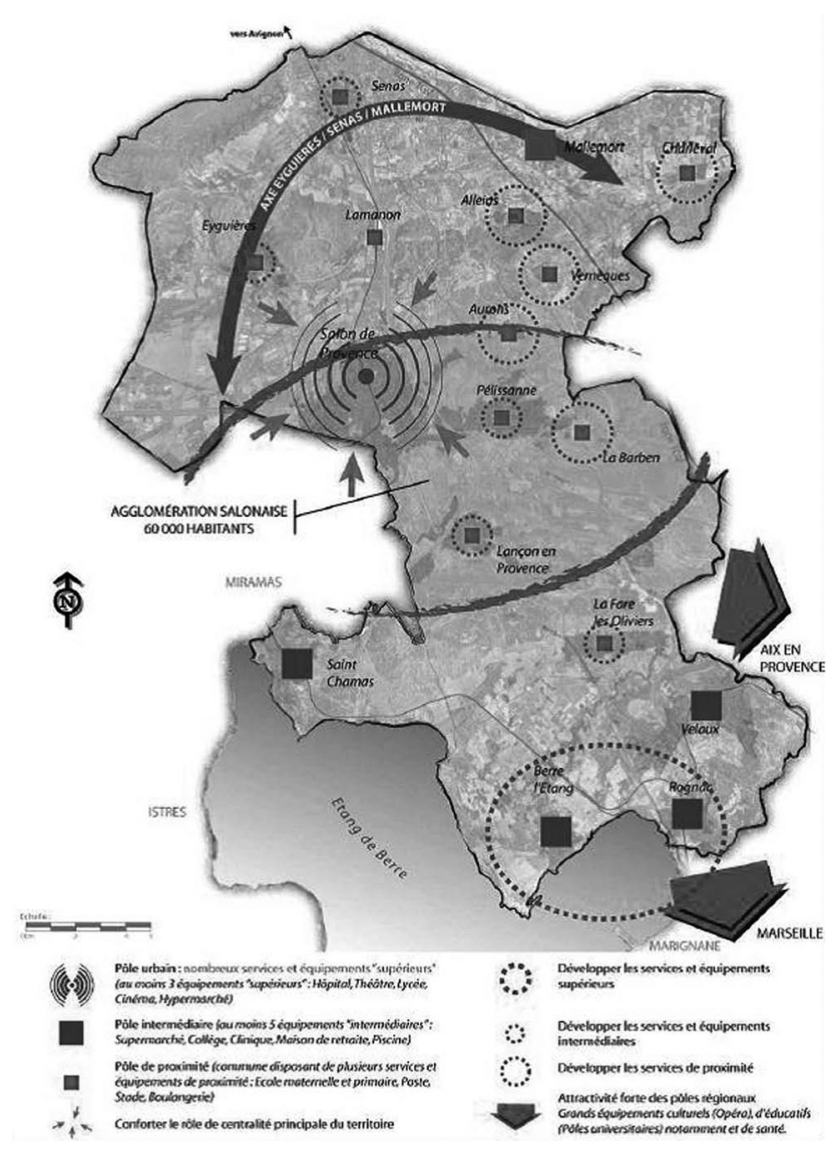

Fig. 3 Le territoire intercommunal tel qu'il est présenté dans le Schéma de cohérence territorial

Source : diagnostic du SCOT, p. 12.

\section{Le SAN-OP : \\ le renversement du consensus communautaire par les pôles secondaires périphériques}

Ici non plus, rien ne prédestinait les communes du SAN-OP à s'associer au sein d'une structure intercommunale. Mais, au milieu des années 1960, l'État décide de créer de toutes pièces un complexe industrialo-portuaire dans le golfe de Fos-sur-Mer et souhaite en faire un pôle de croissance régional. Économiquement, le territoire devient dépendant d'un complexe industrialo-portuaire fait de grandes industries immobiles qui contraignent l'usage du territoire et dont la présence «qualifie » l'espace qu'elles occupent. Ce paysage industriel donne lieu à des représentations qui le connotent d'une manière peu attractive, notamment sur le plan résidentiel, et accueille une proportion importante d'ouvriers et d'employés (Annexe 2.). Pour accompagner l'urbanisation rapide due à l'installation du complexe industrialo-portuaire ${ }^{10}$, l'État crée en 1972 une ville nouvelle. Les communes de Fos-sur-Mer, Istres et Miramas forment un Syndicat communautaire d'aménagement qui devient un Syndicat d'agglomération nouvelle en 1984. Durant 30 ans, l'institution intercommunale ne change pas de périmètre et est dominée par une coalition socialistecommuniste sous un fort leadership de la ville d'Istres, la ville centre. À partir de 2003 et l'entrée de trois nouvelles communes au SAN-OP (Port-Saint-Louis-du-Rhône, Grans et Cornillon-Confoux), le leadership politique de la ville d'Istres est contesté et décline rapidement au profit d'une coalition des pôles secondaires emmenée par Fos-sur-Mer :

Il y a une lutte politique qui existe depuis plusieurs années entre Istres, qui apparaît minoritaire au sein du comité syndical, et les autres communes qui font blocs. Là, on est sur une problématique de : est-ce qu'Istres est la ville centre ou est-ce que l'on est sur [un SAN] qui est multipolaire. Ce que défendent Miramas et Fos, moins Port-Saint-Louis, beaucoup moins Cornillon et Grans bien sûr. Mais ils disent que l'on n'a pas à subir une centralité au niveau d'Istres, centralité qui n'existe pas réellement. ${ }^{11}$

Cette lutte politique s'exprime dans les péripéties qui suivent l'entrée des trois nouvelles communes et la question épineuse de la (re)distribution des sièges du comité syndical. À la suite de leur entrée, une première négociation aboutit à une distribution des 56 sièges qui ne satisfait pas tout le monde, notamment Fos-sur-Mer qui ne détient que $18 \%$ des sièges alors qu'elle procure près de $90 \%$ des recettes fiscales de l'intercommunalité. Ce sujet de désaccord va rapidement tendre les relations entre Istres et Fos-sur-Mer, qui préside l'intercommunalité. Le maire de Fos propose que la répartition des sièges du comité syndical soit pour partie adossée à la taxe professionnelle. Fin 2006, de nouveaux statuts sont adoptés. Le comité syndical compte désormais 71 sièges. Les gagnants sont Fos-sur-Mer, Grans et Port-Saint-Louis, les perdants Istres et dans une moindre mesure Miramas. Cette décision est jugée inacceptable par Istres, qui ne détient plus que $23 \%$ des sièges contre $29 \%$ auparavant. Il faut dire que la modification des statuts du SAN-OP est prise sans les délégués Istréens, lesquels n'étaient pas présents au conseil syndical puisque non encore désignés pour cause d'élections municipales anticipées la semaine précédant la modification des statuts $\mathrm{du}$ SAN-OP. Istres dépose alors un référé au tribunal administratif pour faire annuler la décision. Il s'en suit une exclusion d'Istres du jeu intercommunal : Istres sera privé de

\footnotetext{
${ }^{10}$ La population est multipliée par trois entre 1968 et 2007.

${ }^{11}$ Entretien, directeur des transports, juin 2011.
} 


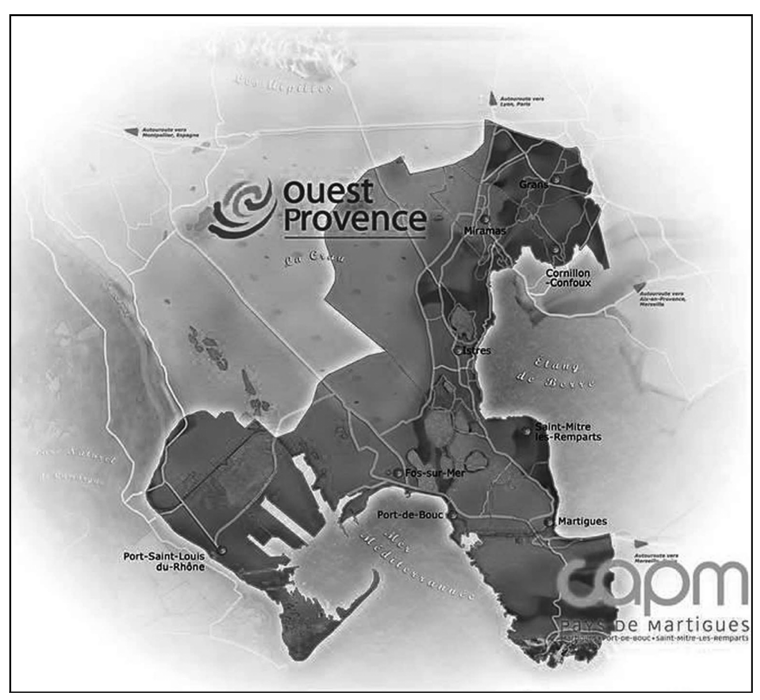

Fig. 4 Une présentation du SAN-OP sur son site internet. La Communauté d'agglomération du pays de Martigues voisine du SAN-OP est aussi représentée sur ce support de communication

Source : http://www.ouestprovence.com/index.php?id=957, consulté le 9 juillet 2010 .

bureau syndical, de vice-présidence et d'un certain nombre de moyens financiers du SAN-OP durant deux ans et demi. Un temps, il sera même envisagé de déménager le siège du SAN-OP d'Istres à Fos.

Dans une institution consensualiste davantage soucieuse $\mathrm{du}$ respect des communes que de l'affirmation ou $\mathrm{du}$ renversement d'un pouvoir politique à l'échelle de l'agglomération [1], cet épisode hors norme, qui casse pendant un moment le consensus communautaire, permet aux autres communes de l'intercommunalité de s'extraire de trente années d'un leadership Istréen et de reprendre les rênes de l'institution. Bien entendu, la situation politique intercommunale reprend un cours «normal » en juin 2009, après la décision du maire d'Istres de retirer l'action en justice de sa commune contre le SAN-OP, néanmoins, cette politisation éphémère de l'arène intercommunale ${ }^{12}$ marque magistralement le passage d'un consensus communautaire (une intercommunalité au service du rayonnement de la centralité Istréenne) à un autre : une intercommunalité au service du développement d'un territoire multipolaire.

\footnotetext{
12 Cette politisation n'est pas issue d'un clivage partisan, les communes d'Istres et Fos sont toutes deux dirigées par des membres du Parti socialiste ou apparentés au Parti socialiste, mais d'un clivage spatial : une coalition de pôles périphériques emmenée par Fos s'oppose à la ville centre.
}

\section{Quand la structure urbaine joue : polarisation versus maillage de la desserte en transport public}

\section{Le choix d'un réseau à deux vitesses dans un espace polarisé}

Le réseau de transport collectif élaboré par l'AgglopoleProvence ${ }^{13}$, dénommé Libébus, est en grande partie créé ex nihilo par la nouvelle administration intercommunale. La densification permanente de l'offre de service du réseau communautaire ces dix dernières années a pour fonction de proposer une alternative à l'automobile et d'engager un report modal dans la ville centre. Sur la période observée, l'intercommunalité élabore une desserte hors de la ville-centre (Figure 5 Annexe 1). Cette desserte des territoires périphériques est nécessaire à la satisfaction des intérêts communaux qui caractérisent l'intercommunalité. Cette politique pourtant significative de desserte des territoires périphériques ne constitue pas pour autant le cœur de l'action communautaire. De fait, l'action intercommunale s'appesantit sur la construction d'une nodalité, et donc la valorisation d'un espace au sein du territoire communautaire : Salon-de-Provence.

Dans un premier temps, les communes périphériques sont desservies par un réseau en étoile à destination de Salon-de-Provence et la qualité du service offert sur chaque ligne est fonction de la densité des liens depuis les communes périphériques vers Salon-de-Provence ${ }^{14}$. Ce choix d'organisation de la desserte permet aux habitants des périphéries de bénéficier d'un réseau de qualité, à condition bien entendu qu'ils aient des pratiques de mobilités les dirigeants vers le centre. En creux se dessinent les perdants du point de vue spatial : les communes périphériques du nord de l'intercommunalité dont les liens avec Salon sont faibles ${ }^{15}$. Dans un deuxième temps, ce critère d'organisation du réseau en étoile va alimenter le consensus en justifiant l'intérêt communautaire d'un développement $\mathrm{du}$ réseau de la ville centre, qui en journée supporte les déplacements des habitants de toute l'intercommunalité du fait sa nodalité dans le réseau périphérique. D’importantes

\footnotetext{
${ }^{13}$ La desserte offerte par le réseau de TC du Smiteeb n'est pas prise en compte ici afin d'alléger la démonstration dans le cadre de l'article.

${ }^{14}$ Les données Insee relatives aux déplacements domicile-travail sont utilisées dès le démarrage du PDU par l'administration communautaire afin de cibler les communes dont la population entretient le plus de liens avec Salon-de-Provence pour leur offrir une plus grande qualité de service.

${ }^{15}$ Moins de $15 \%$ de la population résidant sur le territoire du nord de l'intercommunalité, globalement l'ancienne intercommunalité Collines Durance, effectue des trajets domicile-travail vers Salon-deProvence. Source : Insee, fichier détail Migpro, traitement auteur.
} 
améliorations sont alors apportées au réseau urbain de Salon-de-Provence. Il s'agit du territoire qui regroupe à lui seul $45,3 \%$ des territoires nouvellement desservis par l'intercommunalité entre 1994 et 2011. Ainsi l'ensemble du territoire urbanisé est couvert par une desserte en TC (Figure 5). De plus, le niveau de service offert par le réseau de transport public est sans commune mesure avec ce qu'il est dans les périphéries et avant la mise en place du réseau communautaire (Figure 5 Annexe 1). L'amélioration de la fréquence de desserte, l'extension des plages horaires de fonctionnement du service et la diamétralisation des lignes de TC améliorent nettement le service offert : la fréquence de la desserte est multipliée par deux entre 1994 et 2011. Enfin, le service de Transports à la demande (TAD) mis en place sur la CA-AP ${ }^{16}$ symbolise la valorisation relative d'un espace par la création d'un réseau à deux vitesses du point de vue spatial et social. Les arrêts desservis sont très peu nombreux (20) et localisés dans l'hyper centre historique de Salon-de-Provence d'une part, et dans les ensembles pavillonnaires huppés de l'Est de la ville, d'autre part [19].

Que ce soit pour les TAD ou le réseau conventionnel, la priorité donnée à Salon-de-Provence dans l'organisation $\mathrm{du}$ réseau résulte d'une double contrainte. La première est celle de l'ambition de la politique communautaire : le report modal de la voiture particulière vers des solutions alter-mobiles. La seconde est une contrainte budgétaire. En effet, lors du cadrage de la politique de déplacements l'accent est mis sur la diminution de certains effets néfastes de l'automobile par la promotion de pratiques « alter-mobile » à même de modifier les comportements des individus et d'enrayer l'usage néfaste de l'automobile. Ainsi si l'on en croit le journal intercommunal, la critique et la mobilisation collective à l'encontre de l'automobile est sévère, sans appel et nécessite une modification généralisée des comportements individuels : "Il n'est jamais évident de revenir sur ses habitudes. La voiture est tellement du domaine du réflexe! Mais changer de réflexe changera l'avenir, j'en suis convaincu. C'est un peu comme arrêter de fumer, mieux vaut le faire maintenant que trop tard. » (vice-président délégué au transport dans le dossier « Transports collectifs. Guérir de la voiture?», Magglopôle, $\mathrm{n}^{\circ} 4$, septembre 2009, p. 8).

Cependant, l'acceptation qu'ont les élus et techniciens de la CA-AP de la dépendance automobile est soit celle d'une relation inverse à la densité [20], soit celle d'une addiction à un mode de déplacement. Jamais la dépendance automobile n'est perçue comme un processus engendré par le développement du système automobile [21]. Dès lors, les

\footnotetext{
${ }^{16}$ Selon la classification proposée par Élodie Castex [18], il s'agit d'un service à itinéraire non-contraint de type semi-polarisé d'arrêts à arrêts. C'est-à-dire que l'on peut se rendre d'un ensemble de semis de points périphériques vers un semis de point central.
}

compétences permettant de freiner le système, notamment le stationnement, sont absentes des discours et ne sont pas prises en charge par l'intercommunalité. Ce n'est pas nouveau, l'automobile est, en règle générale, ménagée par les politiques locales de transports et de déplacements, quand bien même elles affichent un enjeu de report modal $[22,16]$. Partant de là, l'amélioration de l'attractivité des transports collectifs doit permettre à elle seule d'opérer un report modal. Rapidement donc, les élus et techniciens placent la ville centre, Salon-de-Provence, comme le lieu où tout se joue ${ }^{17}$. En effet, sur cet espace dense non seulement la rentabilité du réseau est meilleure, puisqu'il existe une demande potentiellement plus importante, mais de plus c'est le lieu où le report modal est possible, puisque l'usage de l'automobile est perçu comme une fonction inverse de la densité. Ce raisonnement satisfait la forte contrainte budgétaire qui pèse sur cette compétence, il permet de réduire les coûts en ne misant que sur un espace : Salon-de-Provence et sa proche périphérie. Bref, modifier massivement les comportements afin d'engager un report modal suppose de miser sur Salon-de-Provence, de « reconquérir la ville de Salon, [et] la rendre au piéton ${ }^{18}$, tout en délaissant les territoires périphériques les plus éloignés aux logiques de mobilités éclatées.

\section{Un réseau pour desservir le «bout du monde » dans un espace multipolaire}

Tout comme l'institution communautaire, la politique de transport collectif du SAN-OP s'inscrit dans le temps long. Dès la fin des années 1970, le Syndicat communautaire d'aménagement élabore pour chacune des communes qui le composent un réseau urbain. En 1990, le réseau se restructure, il est adossé à la desserte des établissements scolaires, et une ligne interurbaine traversant le territoire intercommunal du Nord au Sud est créée. Ce réseau, dont la fonction n'est pas d'engager un report modal mais d'offrir une desserte fine du territoire, sera refondu en 2005 afin de prendre en compte l'arrivée des trois nouvelles communes.

L'évolution de la densité de desserte entre 1999 et 2011 mais aussi la décomposition de la desserte actuelle nous permettent d'observer une politique communautaire axée sur la solidarité spatiale (Figure 6 Annexe 2). Tout d'abord,

\footnotetext{
${ }^{17}$ Lors d'un entretien, un ancien technicien transport de la CA-AP résumait bien le consensus communautaire privilégiant le centre urbain le plus important de l'intercommunalité. Il soutenait que « Les problématiques urbaines, sur l'agglo, elles concernent Salon. [...] Globalement les gens sont conscients que c'est là [à Salon-deProvence] que ça ce passe en matière de transports urbains, et nulle part ailleurs [...]. Sur les autres territoires, on ne peut pas avoir la même approche, on est sur du tissu extensif périurbain. On peut guère faire mieux pour ces communes en termes de report modal. »

${ }^{18}$ Entretien, Vice-président de la CA-AP délégué aux transports.
} 

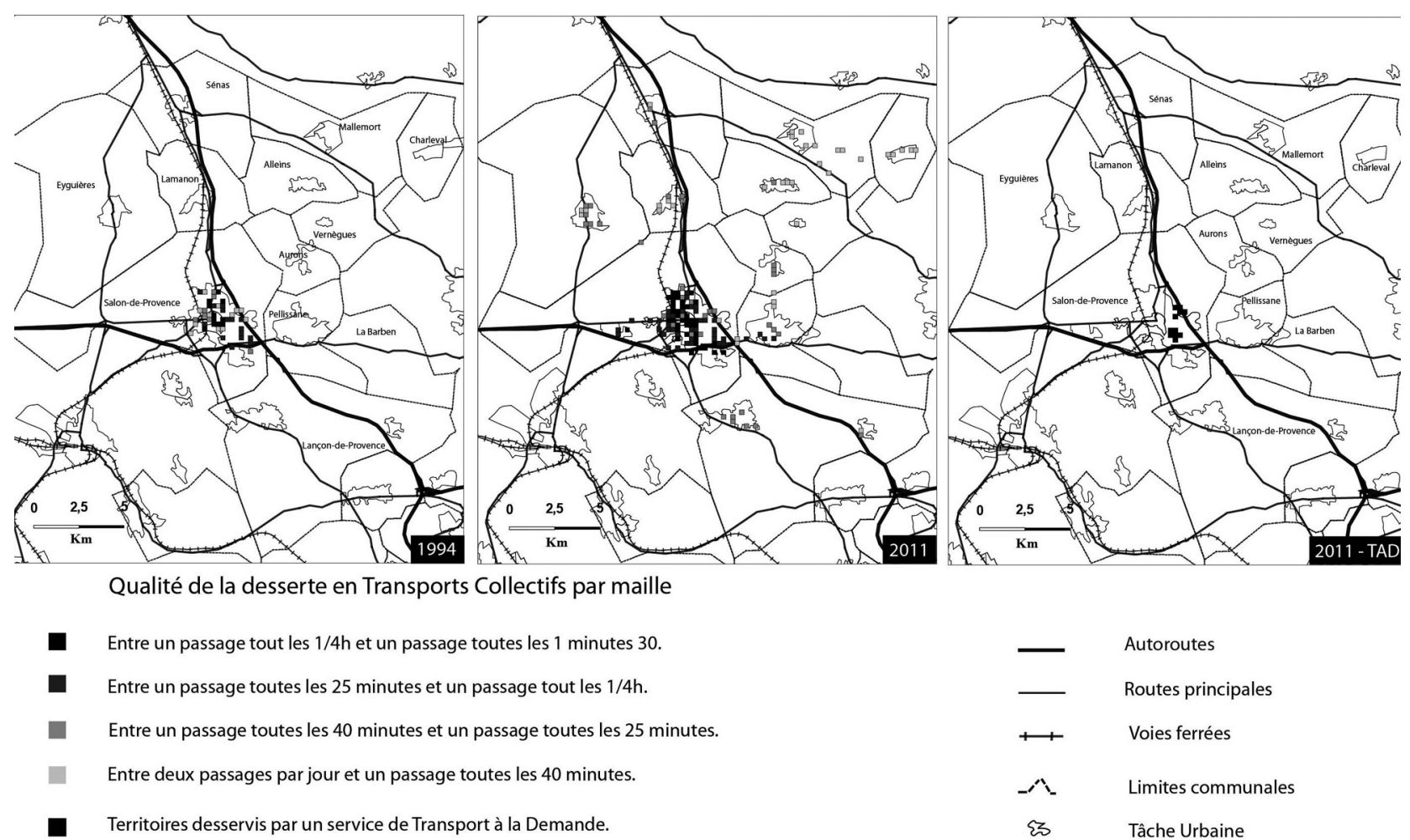

Qualité de la desserte en Transports Collectifs par maille

Entre un passage tout les $1 / 4 \mathrm{~h}$ et un passage toutes les 1 minutes 30.

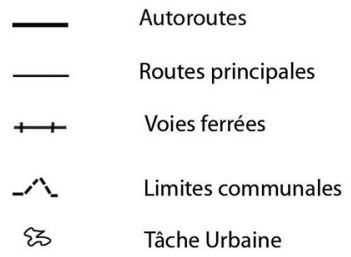

Fig. 5 CA-AP : évolution de la desserte depuis 1994

Source : UE - SOeS, Corine Land Cover, 1990 et 2006, CA-AP, 2009, IGN/PFAR-PACA. Conception et réalisation : M. Claux, 2012.

le fait marquant de la politique communautaire entre 1999 et 2011 est l'effort conséquent réalisé afin de desservir Port-Saint-Louis-du-Rhône, 20,8 \% des territoires investis par le réseau communautaire ces dix dernières années sont situés dans cette petite commune ouvrière excentrée. Cet effort part du constat de l'isolement de cette commune vis-à-vis des territoires alentours :

Les Port-saint-Louisiens, ils n'ont pas de train, ils ont deux lignes par jours vers l'extérieur de leur territoire. Ils sont complètement isolés. [...] Avant, Port-Saint-Louis, ça me faisait penser à la Sibérie. Pas au niveau du climat, mais c'est le bout du monde. Donc, il n'y a pas de gare, il y a un isolement complet. Donc, il fallait rompre cet isolement. ${ }^{19}$

Ensuite, la desserte en transports collectifs est distribuée de manière « homogène » sur le territoire, tant du point de vue des territoires desservis que de la qualité de la desserte. Ainsi, plus de $50 \%$ des mailles bénéficiant d'une desserte supérieure à la médiane intercommunale se situent

\footnotetext{
${ }^{19}$ Entretien, directeur des transports, juin 2011.
}

sur les pôles secondaires de Miramas, Fos-sur-Mer, et dans une moindre mesure à Port-Saint-Louis-du-Rhône. Enfin, le système de TAD met en avant la finesse de la desserte développé par le SAN-OP et contraste avec le service de TAD de la CA-AP. D'une part, ce service s'étend sur l'ensemble du territoire et concerne un nombre d'arrêts (305) qui dépasse largement le nombre d'arrêts desservis par les transports publics conventionnels (188). D'autre part, c'est un service offrant une grande accessibilité au territoire puisqu'il s'agit, à l'usage, d'un système de TAD non contraint d'arrêt à arrêt ou la montée et la descente peut s'effectuer depuis et vers n'importe quel arrêt du territoire intercommunal. Initialement, les TAD ont été pensés pour permettre aux habitants des petites communes de Grans et Cornillon-Confoux d'avoir des moyens de se déplacer, il a finalement été généralisé à l'ensemble du territoire.

Ces éléments relatent la fonction attribuée aux TC par le directeur des transports de l'intercommunalité en poste depuis la création de la ville nouvelle. Selon lui, la politique de transports collectifs a la vocation d'offrir les moyens de se déplacer à un public contraint (les scolaires, les RMIstes, les ménages sans automobile, les personnes en 


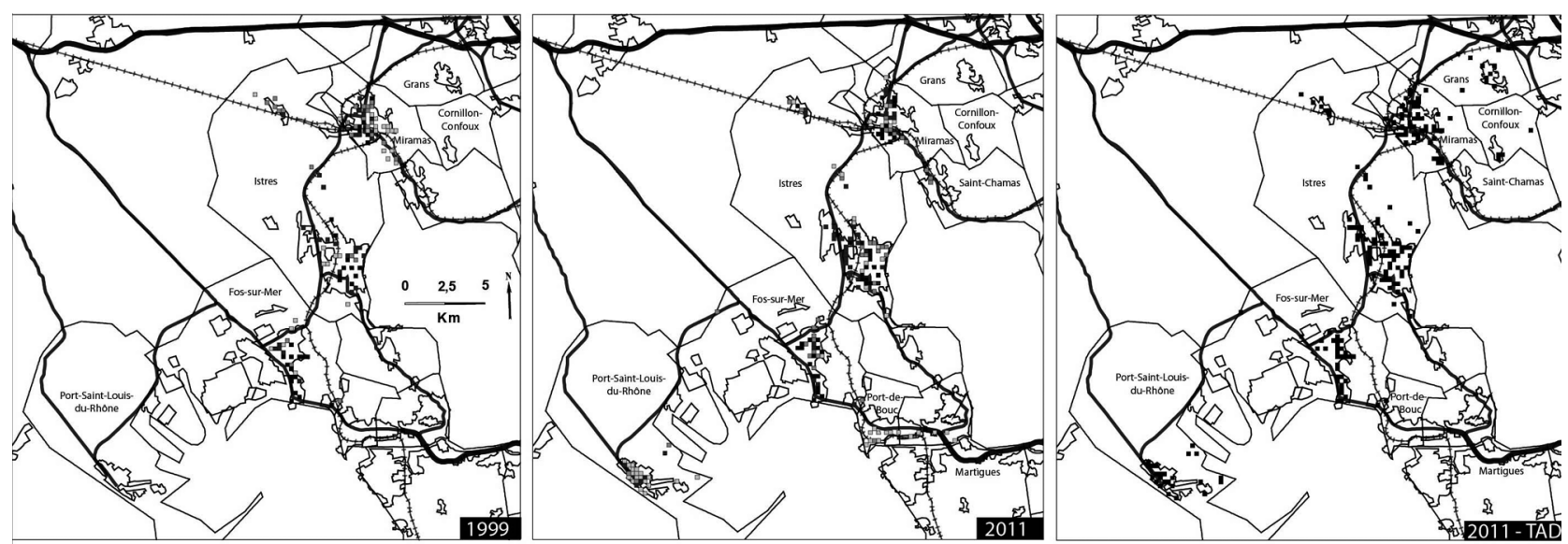

Qualité de la desserte en Transports Collectifs par maille

Entre un passage toutes les 20 minutes et un passage toutes les 6 minutes 30.

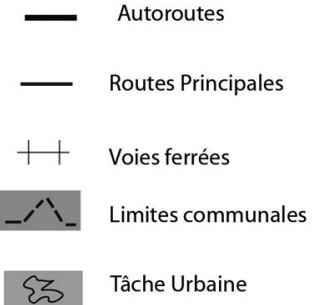

Territoires desservis par un service de Transport à la Demande

Fig. 6 SAN-OP évolution de la desserte

Source : UE - SOeS, Corine Land Cover, 1990 et 2006, SAN-OP, 2010, IGN/PFAR-PACA ; Conception et réalisation : M. Claux, 2012.

démarche d'insertion...). Ce public qui représente presque $80 \%$ des utilisateurs du réseau de transports bénéficie de la gratuité. Dès lors, le critère d'efficacité économique dans les décisions de développement du réseau ne joue plus, c'est le critère d'équité spatiale de la desserte qui prend le pas. Cette politique et ces choix sont favorisés par la présence d'une coalition des pôles secondaires plébiscitant cette stratégie de développement du réseau qui ne cherche pas uniquement à intensifier et étendre la desserte sur la ville centre. Ce qui explique une densité de desserte médiane plus faible que celle observée sur la CA-AP.

Les critères qui guident les choix d'organisation de ces deux réseaux de transports publics sont fortement différenciés et leurs fonctions respectives le sont aussi. Certes, ces fonctions sont liées en partie à la structure urbaine de ces territoires. Tandis que la CA-AP est marquée par une polarité principale, le SAN-OP est un territoire multipolaire. Toujours est-il, qu'au-delà de cette variable, les choix d'organisation des réseaux sont différents. D'un côté, l'ambition d'un report modal associé à la contrainte budgétaire entraîne une polarisation des investissements dans la ville centre. De l'autre, le choix effectué de desservir toutes les populations et tous les territoires d'une manière à peu près similaire amène la desserte en transports collectifs à mailler l'ensemble du territoire. Ces fonctions différenciées des deux réseaux s'observent aussi à travers les projets symboliques étudiés.

\section{Quand la structure sociale joue : mobilités «douces et propres » pour un centre-ville revalorisé versus aide sociale à la mobilité}

\section{Libébulle : l'objet symbolique d'un projet urbain}

En avril 2010, à la demande du maire de Salon-de-Provence et président de l'intercommunalité, le service transport est chargé de mettre en œuvre une nouvelle ligne de transport collectif urbain à destination du centre-ville Salonnais : la navette communautaire Libébulle. Cette navette est un objet standardisé du développement urbain durable développé et mis en œuvre dans le cadre d'une mise en scène du centre-ville de Salon-de-Provence. Cette ligne de bus effectue une boucle de 2,5 kilomètres, et relie entre elles les différentes parties d'un projet urbain communal. Elle part de la gare, futur pôle multimodal doté 
d'un écoquartier, passe par la place Jules-Morgan, où une importante opération urbaine est en cours, transite par les cours récemment rénovés du centre-ville et revient à la gare. La fréquence de la desserte est la plus élevée du réseau, un passage toutes les dix minutes en heure de pointe. La navette est gratuite. Les minibus qui effectuent les trajets fonctionnent à l'énergie électrique et font figurer la mention de véhicule écologique dans une optique de marketing urbain :

Libébulle est un minibus $100 \%$ électrique, avec une absence totale d'émissions de gaz polluants et de gaz à effet de serre, très peu bruyant, contribuant ainsi à la diminution des émissions sonores en centre-ville. ${ }^{20}$

Le choix du véhicule électrique, c'est l'aspect écologique. On s'est dit que ce serait bien que ce soit un véhicule électrique pour l'image, pour la vitrine de la ville. ${ }^{21}$

Les usagers de la navette Libébulle effectuent des trajets courts, inférieurs à 600 mètres. De fait, si Libébulle est bien la première ligne du réseau en termes de voyageurs transportés, elle capte essentiellement des piétons. Ironie du sort, elle effectue donc un report modal de la marche à pied vers les transports collectifs urbains. Certes, la navette Libébulle n'est un pas un objet technique prestigieux. Ce n'est ni un tramway, ni un bus à haut niveau de service. C'est un petit train touristique modernisé par le développement urbain durable ${ }^{22}$. Elle n'a pas autant de capacité que les Transports collectifs en site propre à valoriser les espaces publics. Pour autant, il s'agit d'une question d'échelle. La navette électrique Libébulle est un objet technique adapté à la mise en scène de la qualité urbaine dans une ville moyenne d'une intercommunalité pour laquelle « la qualité de vie, [est] le grand enjeu [du] PDU $»^{23}$. Dans la ville centre gagnante de cette politique communautaire, le centre-ville patrimonial à forte valeur foncière est gagnant. Il est mis en valeur par la politique communautaire. Et, cette mise en valeur du centre patrimonial s'adresse aux populations aisées de la toute proche Provence Salonaise, tant en terme de lieu de consommation que du point de vue du choix résidentiel.

\footnotetext{
${ }^{20}$ Source : http://www.libebus.com/index.php/libebus/se-deplacer/ navette-gratuite-libebulle, consulté le 9 juin 2011. Mise en italique originale.

${ }^{21}$ Entretien, adjointe aux déplacements de la commune de Salon-deProvence, juillet 2012.

22 Durant les périodes estivales, une calèche, « la navette hippomobile », complète la navette Libébulle sur un itinéraire différent.

${ }^{23}$ Vice-Président délégué au PDU, Magglopole, $\mathrm{n}^{\circ} 4$, septembre 2009, p. 11.
}

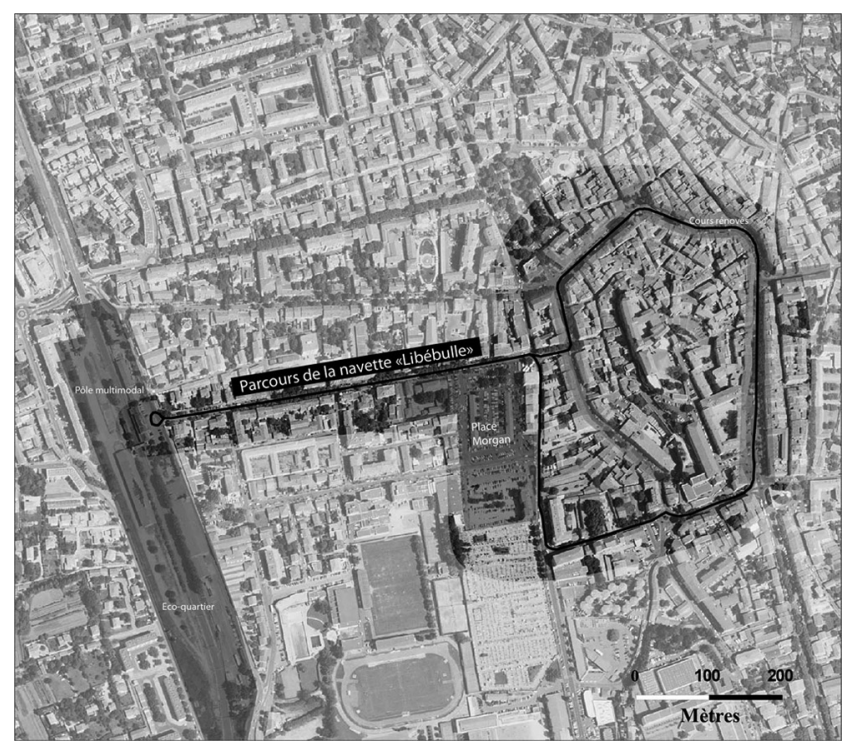

Fig. 7 Navette Libébulle

\section{Des bus vers l'emploi : un projet symbolique à forte teneur sociale}

La problématique de l'accès à l'emploi prend un sens bien particulier sur le territoire de l'ouest de l'Étang de Berre. Durant les décennies 1970 et 1980, les grandes industries qui s'implantent et emploient une part importante de la population disposent toutes d'un service de transports dédié pour emmener leur main-d'œuvre aux usines. Progressivement ces entreprises emploient une part de moins en moins importante de la population, les activités industrielles déclinent puis se diversifient. Or, les nouveaux employeurs industriels, notamment dans le secteur de la logistique, ne mettent pas en place un système de ramassage des employés.

Le développement progressif du secteur logistique sur le territoire fait qu'à partir du milieu de la décennie 2000, la problématique de l'accès à l'emploi et plus largement celle des conditions de mobilité des pauvres s'inscrit à l'agenda communautaire. Au début de l'année 2009, un fonctionnaire du service de la direction de l'emploi et de la cohésion sociale est missionné par le président de l'intercommunalité, afin d'élaborer des propositions opérationnelles sur la problématique des difficultés de mobilités des personnes en parcours d'insertion sociale et professionnelle. Le chargé de mission regroupe différents partenaires institutionnels et réalise un diagnostic partagé sur les besoins de mobilités de ce public cible sur le territoire intercommunal. Une fois encore, c'est par le biais d'un acteur social qu'avance la question sociale de la mobilité. 

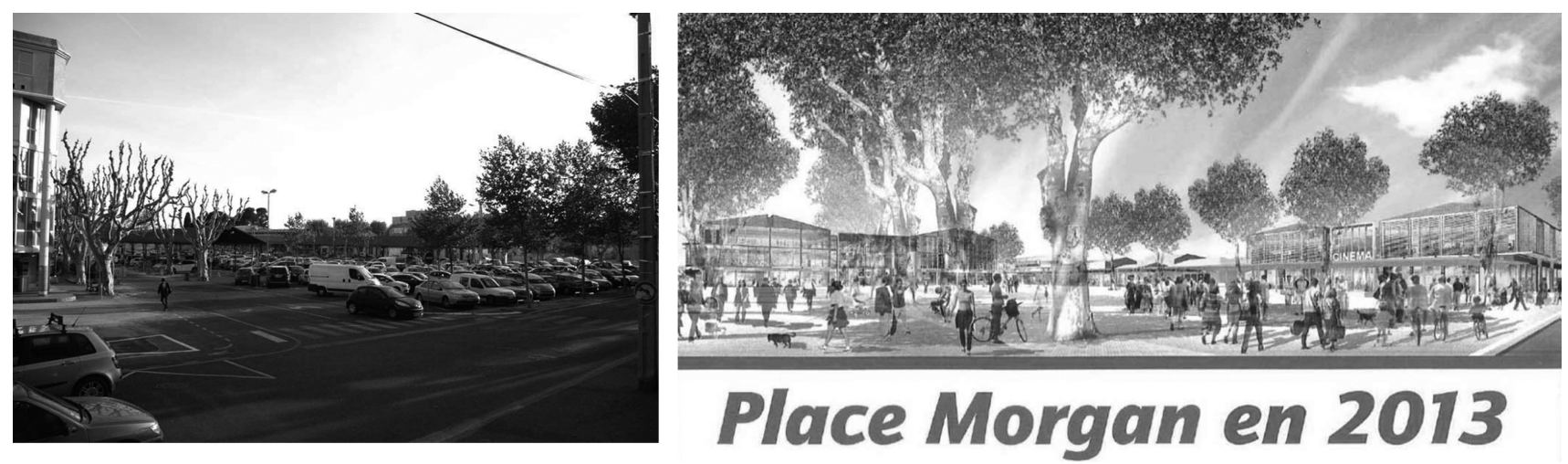

Fig. 8 La place Jules-Morgan actuellement et une vue d'artiste de ce qu'elle sera en 2013

Source : cliché auteur et support de communication municipale : «fais-moi une place ».

À l'issue du diagnostic trois propositions sont élaborées : le développement des TC à destination des pôles logistiques, la création d'une plate-forme de mobilité, et la mise en place d'un garage et d'une auto-école sociale. Seule la première proposition nous intéressera ici, puisqu'elle est la première pierre d'une coopération entre les différentes entreprises logistiques et la puissance publique afin de réaliser un PDIE, qui représente un objectif à moyen terme de l'intercommunalité.

Le développement de l'offre TC à destination des zones logistiques s'effectue via la réalisation de plusieurs lignes de transports collectifs dans le cadre d'un appel à projet de la Délégation Interministérielle à la Ville portant sur la thématique des quartiers vers l'emploi. Si, selon les mots employés par le directeur général adjoint aux transports, il s'agit «d'un véritable service public, d'un service public aux personnes défavorisées » qui offre la possibilité de se déplacer vers son lieu de travail, la direction transports du SAN-OP estime que ces lignes TC ne sont pas adaptées aux spécificités du territoire. Le taux de remplissage de ces navettes est faible, il est parfois de l'ordre de l'unité. Néanmoins, la direction des transports estime qu'il est nécessaire de poursuivre cette action et que l'actuel levier de développement de ce « service public aux personnes défavorisées » réside dans une coordination entre les entreprises, et plus précisément un chrono-aménagement portant sur les heures de prises et de fin de postes. À titre d'exemple, le site logistique de Clésud (700 emplois) connaît 11 horaires différents de prise de poste entre $5 \mathrm{~h}$ et $11 \mathrm{~h}$ du matin, pour seulement deux rotations de TC. Actuellement, des discussions avec les entreprises afin de regrouper les heures de prise et de fin de poste sont en cours mais l'aboutissement de ces négociations reste incertain du fait de la réticence des acteurs privés :
Certaines entreprises, dans la logistique en particulier, n'ont pas envie que se retrouvent dans les mêmes bus leurs personnels. Parce qu'ils peuvent échanger sur leurs conditions de travail, leurs fiches de paye, etc. Et, il y a certainement des entreprises qui n'ont aucune envie que ces informations circulent. Il est plus facile d'avoir des batailles sociales quand les ouvriers se regroupent entre différentes entreprises. C'est un élément social qu'il ne faut pas négliger et qui peut amener des résistances au niveau des entreprises [pour la formalisation de Plan de déplacements inter-entreprises] ${ }^{24}$

\section{Conclusion}

Cette comparaison de deux réseaux communautaires de transports publics, en s'attachant à évaluer les territoires et groupes sociaux gagnants, apporte des résultats qui invitent à dépasser une vision statique de l'action intercommunale selon laquelle les structures communautaires seraient enfermées dans un carcan communaliste et par conséquent incapable de mener des politiques redistributives et planificatrices [1]. Sans remettre en cause le consensus communautaire et ses rouages, ce sont les effets qu'il produit a priori sur les politiques intercommunales qui sont à réévaluer. Certes, d'un point de vue macro il peut sembler que toutes les communes et toutes les populations s'avèrent gagnantes devant ces politiques intercommunales. Néanmoins, en affinant l'évaluation de ces politiques, à travers les choix effectués dans l'organisation des réseaux et à travers leurs projets symboliques, certaines communes et certaines populations apparaissent rapidement plus gagnantes que d'autres. Ce travail n'a pas vocation à définir une fois pour toutes le sens de ces deux politiques communautaires,

$\overline{{ }^{24} \text { Entretien, directeur des transports, juin } 2011 .}$ 




Fig. 9 Les lignes de Transports Collectifs dédiées à la desserte des zones d'activités logistiques

Source : SAN-OP, L'emploi à portée de bus, p. 24.

soumis à des compromis et à des consensus communautaires qui peuvent évoluer comme le montre le cas du SAN-OP. Néanmoins, il ouvre quelques pistes de réflexions sur les liens qui unissent la fonction attribuée aux réseaux de transports publics et les caractéristiques socio-économiques des territoires intercommunaux.

Sur le territoire de tradition bourgeoise où prend place la récente $\mathrm{CA}-\mathrm{AP}$, la fonction du réseau de transport public est avant tout la valorisation de Salon-de-Provence, sous couvert d'attractivité du réseau de transport public et de développement urbain durable. En effet, si « les nœuds de réseaux sont valorisés par leur fonction de mise en relation » [23] alors Salon est l'espace valorisé du réseau communautaire et plus largement d'un réseau métropolitain en cours de constitution. Plus finement, c'est le centre historique patrimonial à forte valeur foncière de ce territoire communal qui est gagnant. En effet, la navette Libébulle participe d'un projet qui ouvre de nouvelles opportunités spéculatives dans les marchés foncier et immobilier du centre-ville. En creux, ces choix d'organisation du réseau déterminent aussi les perdants : les habitants des périphéries lointaines et populaires du nord de l'intercommunalité aux logiques de mobilités éclatées. Finalement, en dehors de la ville centre, la réduction des inégalités sociales et spatiales d'accès à la mobilité n'est pas un élément structurant de la politique communautaire, la qualité de vie en ville l'est davantage.

Sur le territoire de tradition ouvrière du SAN-OP, les choix qui président au développement du réseau de transports public font de ce dernier un facteur de solidarité spatiale à l'échelle du territoire communautaire et vers l'extérieur de ce territoire. Ici ce sont alors les territoires isolés, insulaires qui bénéficient prioritairement des développements du réseau dans une perspective de maillage de la desserte en transport public et afin d'offrir à tous les moyens de se déplacer. Et, ce sont les populations populaires qui sont ciblées en premier lieu : les ouvriers des industries logistiques et les personnes en parcours d'insertion professionnelle. Ici, la réduction des inégalités sociales et spatiales d'accès à la mobilité est un élément fort autour duquel se structurent les choix d'organisation du réseau communautaire.

Ainsi, dans les territoires les plus rouillés la question sociale de la mobilité serait prise à bras-le-corps tandis qu'elle serait progressivement évincée à mesure que l'on approche des territoires plus dorés, dans lesquels la qualité de vie en ville et l'augmentation des valeurs foncières et immobilières seraient prioritaires. Ces résultats tendent donc à nuancer les approches radicales néo-marxistes. En effet, l'influence de l'accroissement de la compétition interurbaine sur les politiques urbaines n'est pas homogène, tous les territoires ne délaissent pas leurs actions redistributives au profit d'une action orientée vers le développement économique. Le territoire, son histoire socio-économique et urbaine, joue un rôle dans la formulation des priorités d'actions de ces politiques urbaines. Néanmoins, dans un contexte d'homogamie des mariages intercommunaux [17], ces résultats questionnent une possible et durable différenciation des trajectoires de développement des intercommunalités d'agglomération en fonction de leurs profils socio-économiques. 


\section{Bibliographie}

1. Desage F Guéranguer D (2011) La politique confisquée. Sociologies des réformes et des institutions intercommunales. Éditions du Croquant, Bellecombe-en-Bauges, $229 \mathrm{p}$

2. Gallez C (2007) Intercommunalités, transports urbains et pouvoir d'agglomération. Cinq trajectoires urbaines. Flux 68:43-61

3. Offner JM (2006) Les plans de déplacements urbains. La Documentation Française, Paris, $78 \mathrm{p}$

4. Orfeuil JP (2008) Une approche laïque de la mobilité. Descartes \& Cie, Paris, $169 \mathrm{p}$

5. Kaufmann V Bergman M.M Joye D (2004) Motility: mobility as capital. International Journal of Urban and Regional Research 28:745-756

6. Fol S (2009) La mobilité des pauvres. Belin, Paris, $208 \mathrm{p}$

7. Rougé L (2007), Inégale mobilité et urbanité par défaut des périurbains modestes Toulousains. EspacesTemps.net, Textuel, 25.04.2007, http://espacestemps.net/document2237.html

8. Wenglenski S (2003) Une mesure des disparités sociales d'accessibilité au marché de l'emploi en Île-de-France. Thèse de doctorat en Urbanisme, Université Paris 12, Paris, 373 p

9. Caubel D (2006) Politique de transports et accès à la ville pour tous ? Une méthode d'évaluation appliquée à l'agglomération lyonnaise. Thèse de doctorat en économie des transports, université Lyon II, Lyon, $375 \mathrm{p}$

10. Le Breton E (2005) Bouger pour s'en sortir : mobilité quotidienne et intégration sociale. Armand Colin, Paris, $235 \mathrm{p}$

11. Féré $\mathrm{C}$ (2011) Concilier accès à la mobilité et mobilité durable: la prise en compte des inégalités d'accès à la mobilité dans les politiques urbaines de l'agglomération lyonnaise. Thèse de doctorat en urbanisme aménagement de l'espace, université Lyon II, Lyon, $411 \mathrm{p}$
12. Béal V (2010) Does neoliberalisation matter? Apports et limites d'une notion montante des urban studies dans la science politique française. Working papers du programme villes \& territoires, Paris, Sciences Po

13. Harvey D (1989) From managerialism to entrepreneurialism: the transformation in urban governance in late capitalism. Geografiska Annaler, Series B, Human Geography 71:3-17

14. Smith N (1996) The new urban frontier. Gentrification and the revanchist city. Routledge, Londres, $227 \mathrm{p}$

15. Reigner H Hernandez F (2007) Les projets des agglomérations en matière de transport : représentations, projets, conflits et stratégie de « détournement » des réseaux. Flux 69:21-34

16. Reigner H Hernandez F Brenac T (2009) Circuler dans la ville sûre et durable : des politiques publiques contemporaines ambiguës, consensuelles et insoutenables. Métropoles 5:42-77

17. Estèbe $\mathrm{Ph}$ (2008) Gouverner la ville mobile. Intercommunalités et démocratie locale. PUF, Paris, 76p

18. Castex E (2007) Le Transport à la Demande (TAD) en France : de l'état des lieux à l'anticipation. Modélisation des caractéristiques fonctionnelles des TAD pour développer les modes flexibles de demain. Thèse de doctorat en Géographie, université d'Avignon et des pays de Vaucluse, Avignon, $423 \mathrm{p}$

19. Insee (2010) Portrait social à l'infra-communal de Salon-deProvence. 30p

20. Newman P Kenworthy J (1989) Cities and automobile dependence. An international Sourcebook. Gower Aldershot, Sidney. 388p

21. Dupuy G (1999) La dépendance automobile. Symptômes, analyses, diagnostic, traitements. Anthropos, Paris, $160 \mathrm{p}$

22. Busson J (2009) L'automobile dans le discours politique : un ménagement équivoque. Pouvoirs Locaux 80:107-112

23. Dupuy G (1993) Géographie et économie des réseaux. L'Espace géographique 22:193-209 


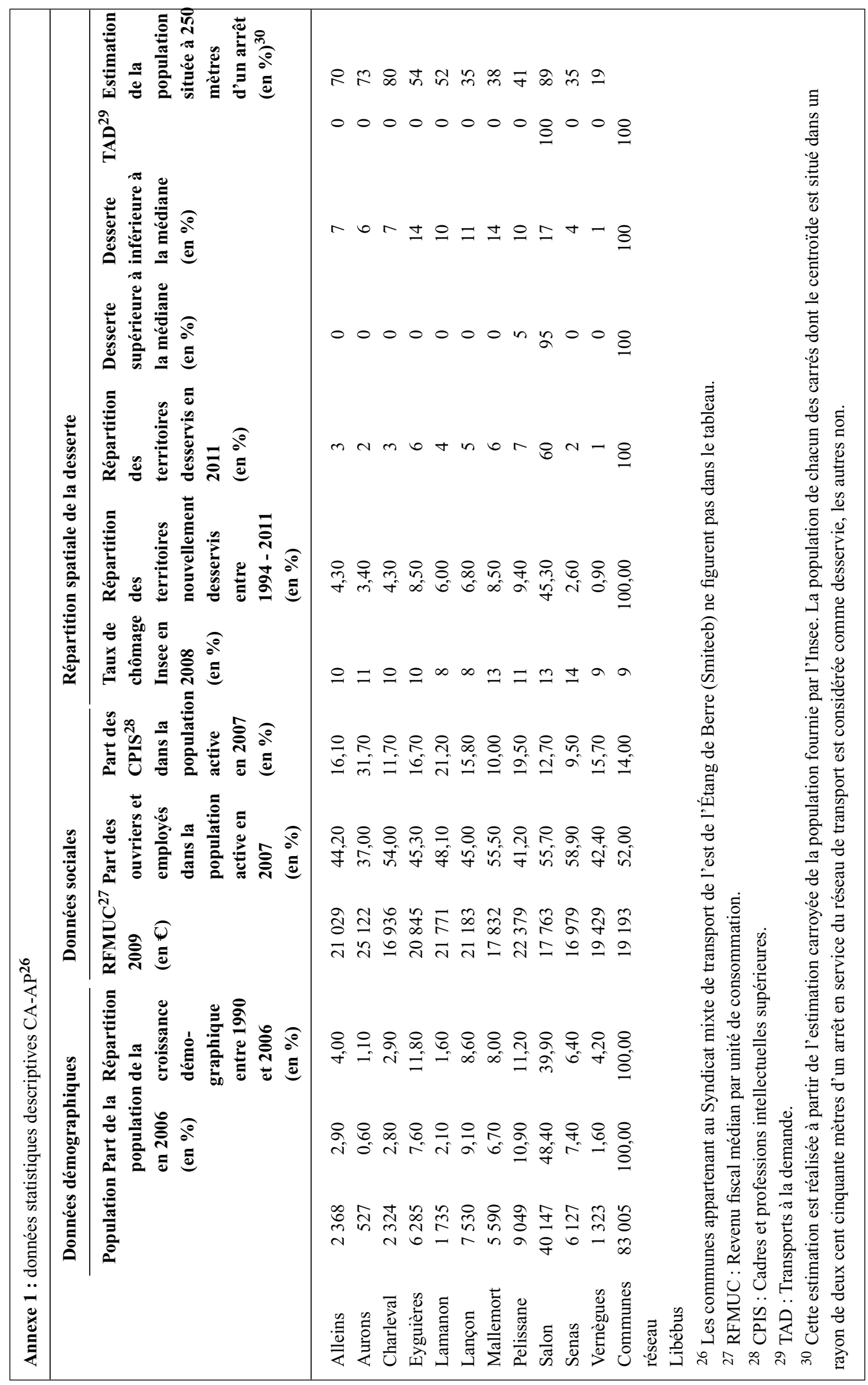

Necplus 


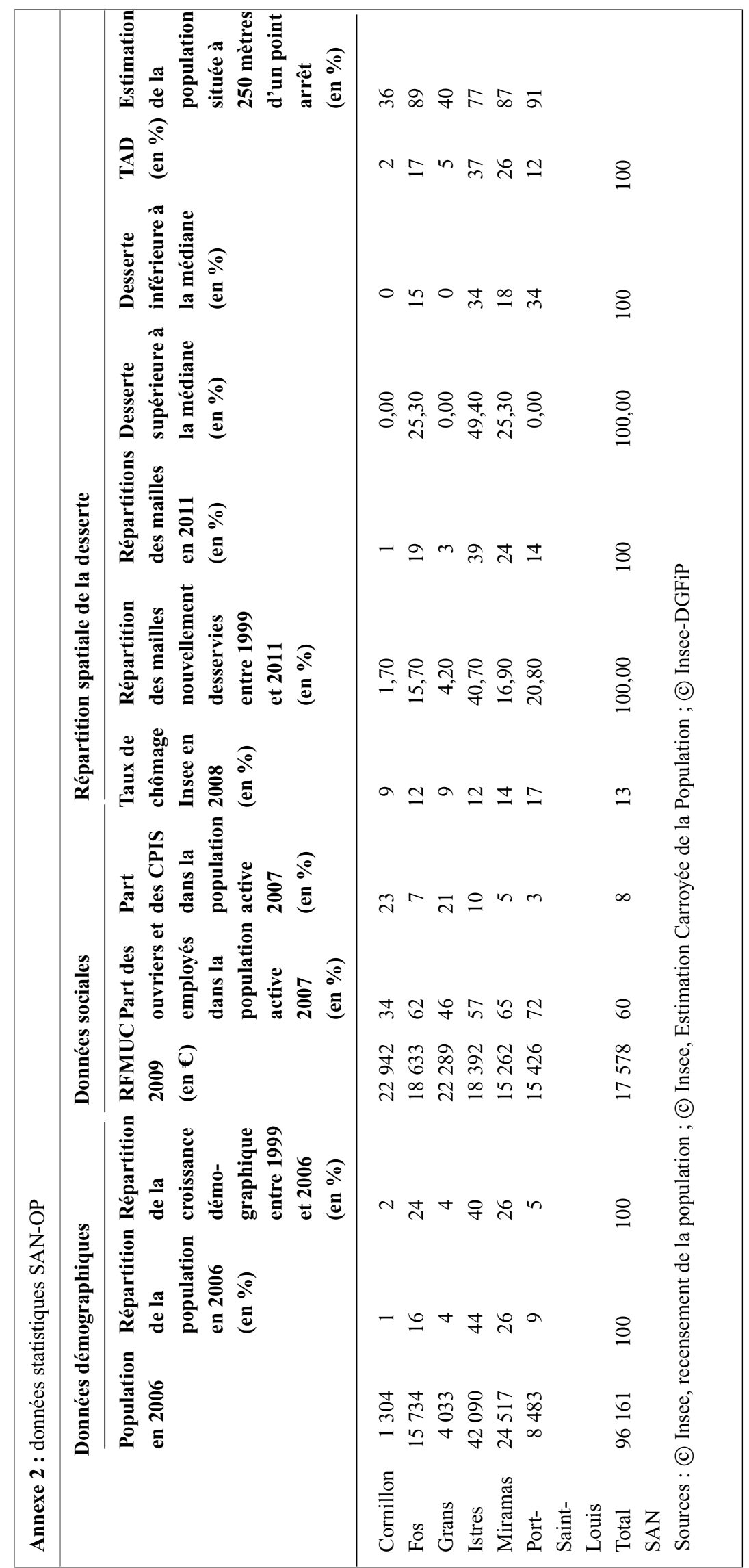

Necplus 